Claremont Colleges

Scholarship@ Claremont

All HMC Faculty Publications and Research

HMC Faculty Scholarship

7-1-1999

\title{
Probing Nonequilibrium Electron Distributions in Gold by Use of Second Harmonic Generation
}

\author{
K. L. Moore' 99 \\ Harvey Mudd College \\ Thomas D. Donnelly \\ Harvey Mudd College
}

\section{Recommended Citation}

K.L. Moore and T.D. Donnelly, "Probing nonequilibrium electron distributions in gold by use of second harmonic generation," Opt. Lett. 24, 990 (1999). doi: 10.1364/OL.24.000990

This Article is brought to you for free and open access by the HMC Faculty Scholarship at Scholarship @ Claremont. It has been accepted for inclusion in All HMC Faculty Publications and Research by an authorized administrator of Scholarship @ Claremont. For more information, please contact scholarship@cuc.claremont.edu. 


\title{
Probing nonequilibrium electron distributions in gold by use of second-harmonic generation
}

\author{
K. L. Moore and T. D. Donnelly \\ Department of Physics, Harvey Mudd College, Claremont, California 91711
}

Received February 18, 1999

\begin{abstract}
Second-harmonic radiation is generated at a gold surface by use of a laser pulse that is varied in duration from 14 to $29 \mathrm{fs}$ and in intensity from $10^{9}$ to $10^{11} \mathrm{~W} / \mathrm{cm}^{2}$. At laser intensities below $10^{10} \mathrm{~W} / \mathrm{cm}^{2}$, the second-harmonic signal has the expected quadratic dependence on pump-laser intensity; however, at higher intensities, the dependence is supraquadratic. This difference arises because the leading edge of the laser pulse interacts significantly with the gold electrons to create a nonequilibrium, photoexcited distribution. The second-harmonic generation process occurs before electron-electron or electron-phonon collisions can equilibrate the distribution and therefore serves as a probe of the nonequilibrium distribution. (c) 1999 Optical Society of America

OCIS codes: $\quad 320.2250,190.4350,160.3900$.
\end{abstract}

The nonequilibrium behavior of many solid-density materials has a time scale of femtoseconds, which was, until recently, too fast to monitor. For example, a great deal of work has been done to understand the form of laser-heated electron distributions in metals at significant time delays after irradiation (see Refs. 1-10, for example), but little has been done to track their evolution from the photoexcited non-Fermi-Dirac distribution to a thermalized, hot Fermi-Dirac (FD) distribution. In past experimental studies the time resolution was no better than $100 \mathrm{fs}$ and significantly worse in most cases (the excellent work by Petek and co-workers, ${ }^{11,12}$ done with an $\sim 10$-fs time resolution, is an exception). However, understanding the form of nonequilibrium distributions and their rapid evolution to thermalized states is of fundamental interest. It is this evolution that constrains theories of electronelectron and electron-phonon scattering dynamics, controls chemical reactions ${ }^{13}$ and instigates macroscopic processes such as melting. ${ }^{14-17}$

In modeling the ultrafast properties of metals the common practice is to assume that a photoexcited electron distribution is instantaneously thermalized, ${ }^{1,3,5-7}$ followed by relaxation with the lattice, according to the theory of Anisimov et al. ${ }^{9}$ This practice is known to be incorrect at short time scales ${ }^{2}$; however, few data exist to help improve our understanding of the system at early times after irradiation or to point the way to development of appropriate models. Using second-harmonic generation (SHG), we investigate the form of the nonequilibrium electron distribution that evolves during photoexcitation by an ultrashort laser pulse. This technique can be extended by use of a pump-probe geometry to measure the electron distribution as it evolves from a nonequilibrium to a thermal distribution.

A nonequilibrium electron distribution can be produced when a metal is irradiated by a nonionizing ultrafast laser pulse $\left(<10^{12} \mathrm{~W} / \mathrm{cm}^{2}\right)$. Modifications that are thus induced in the distribution evolve rapidly owing to the high electron density. In gold, for example, the high-energy tail of a distribution relaxes in less than $50 \mathrm{fs},{ }^{1,3,5,6}$ and the entire distribution equilibrates in approximately $500 \mathrm{fs}^{1-3}$ Electron thermalization with the underlying lattice is slower, occurring on a 1-ps time scale. ${ }^{1-3}$ A sub-50-fs laser pulse can, therefore, be used to measure the photoexcited electron distribution and its evolution from a photoexcited, non-FD distribution to a thermalized, FD distribution.

We make use of this fact in an experiment in which ultrashort laser pulses are used to produce secondharmonic light from a gold surface. The SHG process is a more-sensitive probe of the electron distribution than either linear absorption or reflection because it samples the electron distribution twice. ${ }^{5}$ Further, it is less costly and less complex than the techniques used in photoemission studies. Gold was chosen for our studies for a number of reasons: Its band structure is well studied and understood ${ }^{18,19}$; its small electronic heat capacity means that the deposition of small amounts of laser energy can result in significant perturbations in the electron energy distribution; and its relevant relaxation and thermalization rates have been studied, ${ }^{1,3,4}$ as have its heat-transport properties. ${ }^{20}$ Further, SHG at a gold surface has been used to investigate plasmon resonances ${ }^{21}$ and has been modeled for pump lasers of long duration..$^{5,22,23}$

We use a single pulse to both pump and probe the electron distribution and thus to gather information about the initial photoexcited distribution. A 100-nmthick polycrystalline gold surface is irradiated with transform-limited laser pulses of 14-29-fs duration originating from a Ti:sapphire oscillator with an operating wavelength of $800 \mathrm{~nm}$. We vary pulse durations by changing the positions of the intracavity prisms, and the transform-limited nature of the pulse is preserved with a prism-pair compressor. The pulse durations are measured with an interferometric autocorrelator. The angle of incidence is $45^{\circ}$, and the irradiating light is $p$ polarized and focused to a $5 \mu \mathrm{m}$ by $8 \mu \mathrm{m}$ spot by an off-axis parabolic mirror. At all times the pump-laser intensity remains well below the damage threshold for gold, and care is taken to ensure that none of the measured second-harmonic signal originates upstream of the gold surface. The second-harmonic light is collected with a collimating 
lens and detected with a photomultiplier tube; appropriate blue glass and interference filters are used to remove the $800-\mathrm{nm}$ light, which is collinear with the second harmonic.

At pump-laser intensities below approximately $10 \mathrm{GW} / \mathrm{cm}^{2}$ we observe the expected quadratic dependence $\left(I_{2 \omega} \propto I_{\omega}{ }^{2}\right)$ of the second-harmonic yield on the pump-pulse intensity. However, as the pump intensity is increased and the electron distribution is significantly modified through photoexcitation, the dependence becomes stronger than quadratic, as shown in Fig. 1. A similar result was reported by Papadogiannis and Moustaizis ${ }^{8}$; however, given their pump-pulse duration (450 fs) and electron-electron and electron-lattice equilibration rates in gold ( $\sim 500$ fs and $\sim 1 \mathrm{ps}$, respectively), their results cannot be interpreted as resulting from a nonthermalized electron distribution; the electron distribution that they measured strongly interacted with itself and the underlying lattice. Our results, on the other hand, arise solely through interaction of the laser pulse with the nascent electron distribution; the similarity of the results is not understood at this time.

Note in Fig. 1(a) that the dependence of the secondharmonic yield on pump-pulse intensity does not change significantly between the 14- and the 29-fs pump pulses. This indicates that the portion of the nascent electron distribution that is probed does not thermalize significantly on a $30-\mathrm{fs}$ time scale, which is consistent with the scaling of electron-scattering rates predicted by Landau-Fermi liquid theory, ${ }^{24}$ the lifetime measurements of copper by Ogawa et al. ${ }^{11}$ and extrapolation of the data of Fann et al. ${ }^{3}$ and Sze and co-workers. ${ }^{25,26}$ Thus our laser pulses are sufficient to probe the relevant energy domain of the nascent distribution.

We developed a model of the electron distribution interacting with an ultrafast laser pulse; results for a 29-fs pump pulse are compared with data in Fig. 1(b). To calculate the magnitude of the expected secondharmonic signal we first determine modifications of the electron distribution owing to photoexcitation at each time step during the pulse. For a constant density of states the photoexcited distribution is calculated according to ${ }^{1}$

$$
\begin{aligned}
N_{n+1}\left(\epsilon_{i}\right)= & N_{n}\left(\epsilon_{i}\right)+n_{\gamma}\left(I_{\omega}\right)\left\{\rho_{n}\left(\epsilon_{i}-\hbar \omega\right)\left[1-\rho_{n}\left(\epsilon_{i}\right)\right]\right. \\
& \left.-\rho_{n}\left(\epsilon_{i}\right)\left[1-\rho_{n}\left(\epsilon_{i}+\hbar \omega\right)\right]\right\}
\end{aligned}
$$

where $N_{n}\left(\epsilon_{i}\right)$ is the number of electrons in the $i$ th energy bin (energy between $\epsilon_{i}$ and $\epsilon_{i}+\Delta \epsilon$ ) at time $t_{n}$, $n_{\gamma}$ is proportional to the number of photons absorbed by the distribution between times $t_{n}$ and $t_{n+1}$, and $\rho_{n}\left(\epsilon_{i}\right)$ is the electron probability distribution at time $t_{n}$. Modifications of the electron distribution of gold that were generated by a 29 -fs pulse with an intensity of $1 \times 10^{11} \mathrm{~W} / \mathrm{cm}^{2}$ are shown in Fig. 2 . We assume an initial FD distribution at $300 \mathrm{~K}$, and the density-ofstates function is taken from Ref. 18. Figure 2 shows changes in the distribution owing to photoexcitation and displays the difference between the FD distribution into which the photoexcited distribution will relax and the initial distribution. The inset shows detail of the photoexcited distribution; modifications that are due to two- and three-photon absorption are evident.

Knowing the form of the electron distribution, one can calculate the number of second-harmonic photons that are generated per unit time, $W_{2 \omega}$, from expressions in the literature. ${ }^{23,27}$ We are interested primarily in the change in the number of second-harmonic photons generated as a function of pump-laser intensity. If one knows the modifications to the electron distribution, changes in $W_{2 \omega}$ can be accounted for by an appropriate statistical factor ${ }^{8,23,27}$ :

$$
\begin{aligned}
W_{2 \omega}\left(t_{n}\right) \propto & \int_{0}^{\infty} f\left(\epsilon, t_{n}\right)\left[1-f\left(\epsilon+\hbar \omega, t_{n}\right)\right] \\
& \times\left[1-f\left(\epsilon+2 \hbar \omega, t_{n}\right)\right]\left[1-f\left(\epsilon, t_{n}\right)\right] \mathrm{d} \epsilon,
\end{aligned}
$$

where $f(\epsilon)$ is the electron energy distribution [distinguished from $f\left(\epsilon, T_{e}\right)$, a thermalized FD distribution]. Recall that $f(\epsilon)$ is the probability of finding an electron between energy $\epsilon$ and $\epsilon+\mathrm{d} \epsilon$. The validity of relation (2) does not depend on the system's being thermalized. After the appropriate photoexcited distribution has been determined at each time step the weighting factor is calculated; we integrate this algorithm over the pulse to determine the relative amount of second harmonic that is created.

A comparison of the prediction of our model with data is shown in Fig. 1(b); our simplified model of the electron-photon interaction largely accounts for the

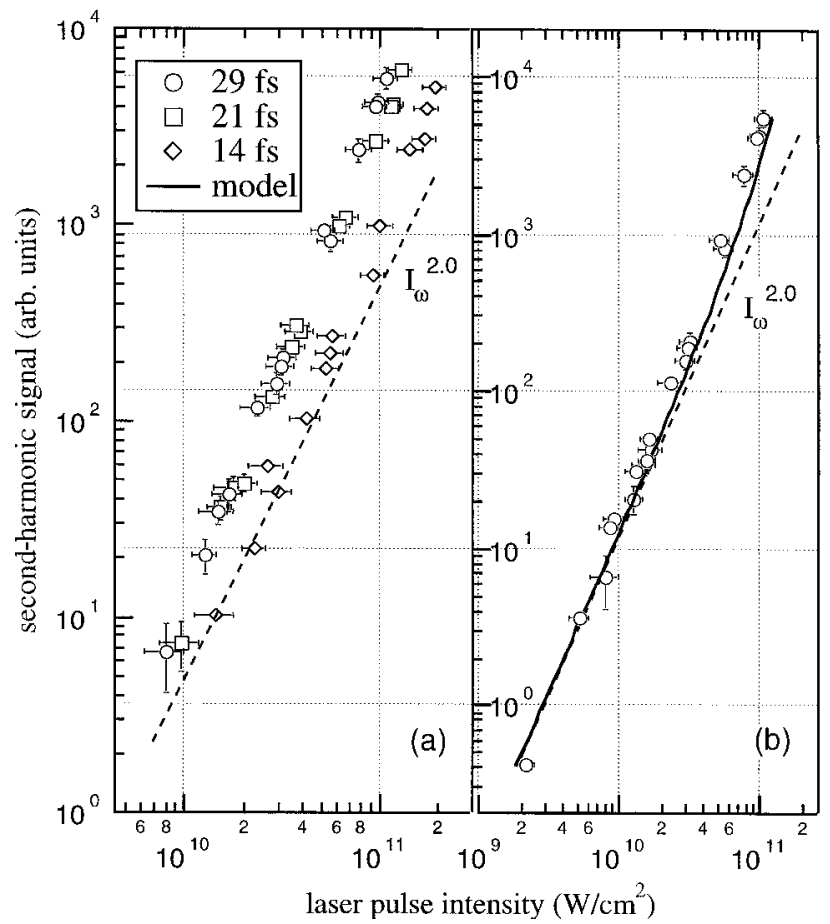

Fig. 1. Second-harmonic yield as a function of peak pumplaser intensity: (a) Supraquadratic dependence of the harmonic signal on pump-laser intensity is measured at each pulse duration. The data indicate that the electron distribution has not relaxed on a $30-\mathrm{fs}$ time scale. (b) Secondharmonic signal plotted against model predictions for a 29 -fs pulse. Note the expanded scales in (b) compared with (a). 


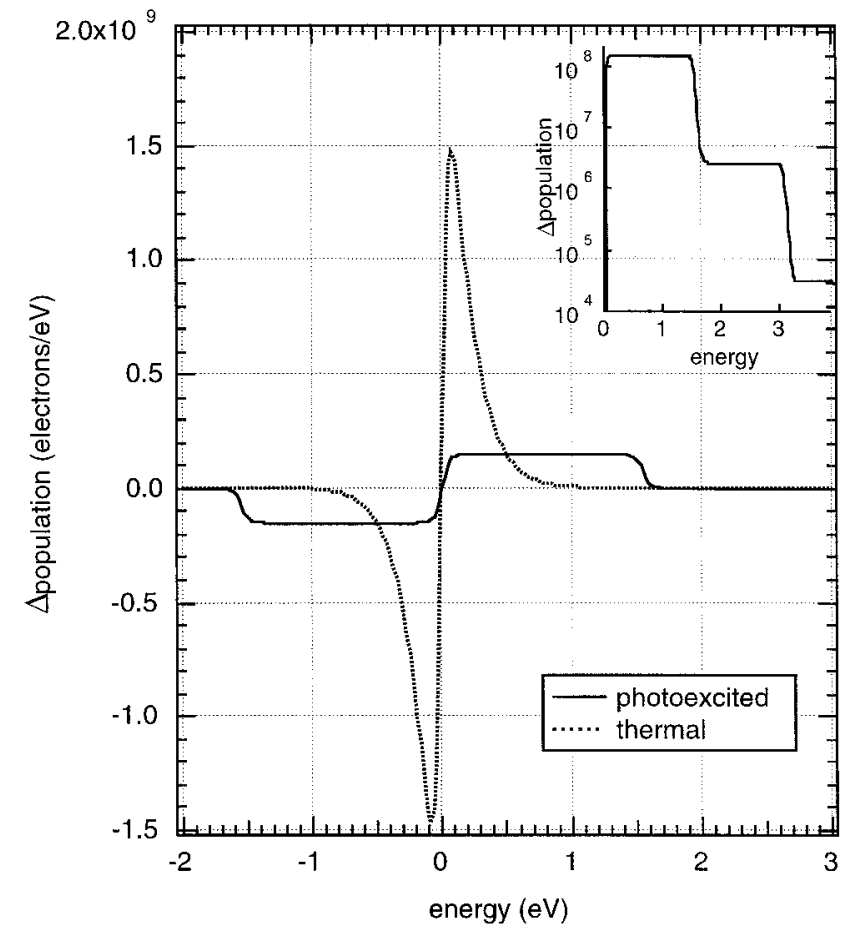

Fig. 2. Difference in population between the nonequilibrium, photoexcited distribution and the initial, thermalized FD distribution. The nonequilibrium distribution relaxes to a thermalized distribution with a temperature of $1570 \mathrm{~K}$; the difference between this distribution and the initial distribution is also shown (dotted curve). The Fermi energy ( $5.5 \mathrm{eV}$ in gold) corresponds to zero energy, and all calculations are for a 29 -fs pulse with an intensity of $1 \times 10^{11} \mathrm{~W} / \mathrm{cm}^{2}$. Inset, detail of the photoexcited distribution for energy greater than zero.

behavior of the second-harmonic signal in both the magnitude and the onset intensity of its departure from quadratic dependence. In this calculation the effective volume into which laser energy is deposited is determined by a single skin depth of $0.02 \mu \mathrm{m}$, the laser spot size, a dynamically calculated reflectivity, and the $45^{\circ}$ angle of incidence. We assume that the initial temperature of the FD distribution is $300 \mathrm{~K}$. The only free parameter in our model is the absolute magnitude of the second-harmonic signal.

In summary, we have shown that an observed supraquadratic dependence of second-harmonic light on pump-laser intensity can be accounted for by knowledge of the form of the time-zero, photoexcited electron distribution. The model that we have developed correlates the magnitude of SHG with this nonequilibrium distribution, and therefore SGH is shown to be a useful tool for understanding the form of nonequilibrium electron distributions. We note that true pump-probe experiments would allow the electron distribution's evolution to be tracked as it relaxes to a thermalized state.

We gratefully acknowledge helpful conversations with P. Saeta, T. Ditmire, and T. E. Glover. This work was supported by research grants from Harvey Mudd College, and the donors of the Petroleum Research Fund, administered by the American Chemical Society, are thanked for partial support of this research. T. D. Donnelly's e-mail address is tom donnelly@hmc.edu.

\section{References}

1. C. K. Sun, F. Vallee, L. H. Acioli, E. P. Ippen, and J. G. Fujimoto, Phys. Rev. B 50, 15337 (1994).

2. W. S. Fann, R. Storz, H. W. K. Tom, and J. Bokor, Phys. Rev. B 46, 13592 (1992).

3. W. S. Fann, R. Storz, H. W. K. Tom, and J. Bokor, Phys. Rev. Lett. 68, 2834 (1992).

4. X. Y. Wang, D. M. Riffe, Y. S. Lee, and M. C. Downer, Phys. Rev. B 50, 8016 (1994).

5. T. A. Luce, W. Hubner, and K. H. Bennemann, Z. Phys. B 102, 223 (1997).

6. J. P. Girardeau-Montaut and C. Girardeau-Montaut, Phys. Rev. B 51, 13560 (1995).

7. J. Hohlfeld, U. Conrad, and E. Matthias, Appl. Phys. B 63, 541 (1996).

8. N. A. Papadogiannis and S. D. Moustaizis, Opt. Commun. 137, 174 (1997).

9. S. I. Anisimov, B. L. Kapeliovich, and T. L. Perel'man, Sov. Phys. JETP 39, 375 (1974).

10. J. H. Bechtel, W. L. Smith, and N. Bloembergen, Phys. Rev. B 15, 4557 (1977).

11. S. Ogawa, H. Nagano, and H. Petek, Phys. Rev. B 55, 10869 (1997).

12. H. Petek, A. P. Heberle, W. Nessler, H. Nagano, S. Kubota, S. Matsunami, N. Moriya, and S. Ogawa, Phys. Rev. Lett. 79, 4649 (1997).

13. J. Phys. Chem. A 102(23), 4021 (1998).

14. C. V. Shank, R. Yen, and C. Hirlimann, Phys. Rev. Lett. 51, 900 (1983).

15. H. W. K. Tom, G. D. Aumiller, and C. H. Brito-Cruz, Phys. Rev. Lett. 60, 1483 (1988).

16. A. H. Chin, R. W. Schoenlein, T. E. Glover, P. Balling, W. P. Leemans, and C. V. Shank, in Ultrafast Phenomena XI, T. Elsaesser, J. G. Fujimoto, D. A. Weirsma, and W. Zinth, eds. (Springer, New York, 1998), pp. 401-403.

17. R. Jimenez, C. Rose-Petruck, T. Guo, K. R. Wilson, and C. J. P. Barty, in Ultrafast Phenomena XI, T. Elsaesser, J. G. Fujimoto, D. A. Weirsma, and W. Zinth, eds. (Springer, New York, 1998), pp. 404-406.

18. R. Lasser, N. V. Smith, and R. L. Benbow, Phys Rev. B 24, 1895 (1981).

19. M. Theye, Phys. Rev. B 2, 3060 (1970).

20. S. D. Brorson, J. G. Fujimoto, and E. P. Ippen, Phys. Rev. Lett. 59, 1962 (1987).

21. D. Steinmuller-Nethl, R. A. Hopfel, A. Leitner, F. R. Aussenegg, and A. Wokaun, Appl. Phys. A 57, 261 (1993).

22. A. T. Georges, Phys. Rev. B 51, 13735 (1995).

23. A. T. Georges, Phys. Rev. A 54, 2412 (1996).

24. D. Pine and P. Nozieres, The Theory of Quantum Liquids (Benjamin, New York, 1966).

25. S. M. Sze, C. R. Crowell, G. P. Carey, and E. E. Labate, J. Appl. Phys. 37, 2690 (1966).

26. S. M. Sze, J. L. Moll, and T. Sugano, Solid State Electron. 7, 509 (1964).

27. S. Varro and F. Ehlotsky, Phys. Rev. A 49, 3106 (1994). 